\title{
Mapping Photoemission and Hot-Electron Emission from Plasmonic Nanoantennas
}

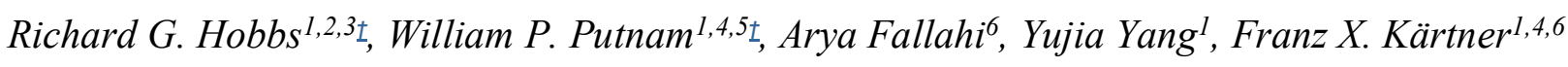
and Karl K. Berggren ${ }^{1}$

${ }^{1}$ Research Laboratory of Electronics, Massachusetts Institute of Technology, Cambridge, MA, U.S.A.

${ }^{2}$ Centre for Research on Adaptive Nanostructures and Nanodevices (CRANN) \& Advanced Materials Bio-Engineering Research Centre (AMBER), Trinity College Dublin, Dublin 2, Ireland

${ }^{3}$ School of Chemistry, Trinity College Dublin, Dublin 2, Ireland

${ }^{4}$ Department of Physics and Center for Ultrafast Imaging, University of Hamburg, Hamburg, Germany.

${ }^{5}$ Northrop Grumman Corporation, NG Next, Redondo Beach, CA, USA

${ }^{6}$ Center for Free-Electron Laser Science, Deutsches Elektronen-Synchrotron, Hamburg, Germany

\pm These authors contributed equally to this work. 
*To whom correspondence should be addressed: Tel: +353 1896 3035; E-mail: hobbsr@tcd.ie

Understanding plasmon-mediated electron emission and energy transfer on the nanometer length scale is critical to controlling light-matter interactions at nanoscale dimensions. In a highresolution lithographic material, electron emission and energy transfer lead to chemical transformations. In this work, we employ such chemical transformations in two different highresolution electron-beam lithography resists, poly(methyl methacrylate) (PMMA) and hydrogen silsesquioxane (HSQ), to map local electron emission and energy transfer with nanometer resolution from plasmonic nanoantennas excited by femtosecond laser pulses. We observe exposure of the electron-beam resists (both PMMA and HSQ) in regions on the surface of nanoantennas where the local field is significantly enhanced. Exposure in these regions is consistent with previously reported optical-field-controlled electron emission from plasmonic hotspots as well as earlier work on low-electron-energy scanning probe lithography. For HSQ, in addition to exposure in hotspots, we observe resist exposure at the centers of rod-shaped nanoantennas in addition to exposure in plasmonic hotspots. Optical field enhancement is minimized at the center of nanorods suggesting that exposure in these regions involves a different mechanism to that in plasmonic hotspots. Our simulations suggest that exposure at the center of nanorods results from the emission of hot electrons produced via plasmon decay in the nanorods. Overall, the results presented in this work provide a means to map both optical-field-controlled electron emission and hot-electron transfer from nanoparticles via chemical transformations produced locally in lithographic materials. 
KEYWORDS: nanoantennas, hot electrons, photoemission, plasmonics, charge-transfer, plasmon decay

Plasmonic nanoparticles provide an efficient means to funnel optical radiation and energy to nanoscale volumes. This ability to couple free-space light to the nanoscale enables dramatically enhanced light-matter interactions that are desirable for optoelectronics and photochemistry applications. ${ }^{1-5}$ As a result, plasmonic nanoparticles have been exploited for sensor technologies, ${ }^{6,7}$ near-field imaging ${ }^{8,9}$ and photovoltaics $^{10,11}$ as well as for photocatalysis, ${ }^{12-15}$ photoluminescence, ${ }^{16-18}$ and even cancer therapy; ${ }^{19}$ more recently, their application space has been further extended to ultrafast electron emitters. ${ }^{20-25}$

Ultrafast electron emission from plasmonic nanoparticles is achieved by exciting the particles' localized surface plasmon resonances (LSPRs) with intense femtosecond laser pulses. The excited surface plasmons generate strong, localized optical fields, which can drive large photoemission currents from the particles. When the magnitude of the local optical field on metal nanoparticles reaches $\sim 10 \mathrm{GVm}^{-1}$ the photoemission mechanism enters a 'field-driven' or tunneling emission regime (this threshold roughly applies to optical fields in the near-infrared). In this strong-field regime, the photoemission process resembles quasi-static field-emission ${ }^{25}$ and (depending on the wavelength and intensity of the driving femtosecond laser pulse) emitted electrons can gain kinetic energies $>10 \mathrm{eV} .{ }^{26}$ As well as driving electron emission, the excited LSPRs can decay on ultrafast timescales to produce energetic, i.e. 'hot', carriers within the metal. ${ }^{27,28}$ These hot carriers can subsequently transfer from nanoparticles to neighboring materials where they can initiate electronic and chemical processes. Control and measurement of such plasmon-induced hot carriers may provide a means to guide photochemical reactions, ${ }^{29-32}$ study the electronic structures of materials, ${ }^{32}$ drive local heating on the nanoscale, ${ }^{19,33,34}$ or harvest energy from light. ${ }^{30}$ 
Mapping field-driven electron emission and hot-carrier-mediated chemical processes on plasmonic nanoparticles will be key to engineering plasmonic structures for ultrafast optoelectronics and hot-carrier-driven plasmonic photocatalysts. Moreover, identifying and differentiating between electronic processes occurring within individual plasmonic nanoparticles will allow for greater control of reaction pathways on their surfaces. In this work, we demonstrate that two common electron-beam lithography resists, poly(methyl methacrylate) (PMMA) and hydrogen silsesquioxane (HSQ), can be used to image both electron emission and energy transfer on the surface of plasmonic nanoparticles.

PMMA has been used in earlier work as an imaging layer for electrons. For example, a PMMA layer was integrated on the anode of a vacuum diode device to image electrons emitted by DC field emission from $\mathrm{ZnO}$ nanowires. ${ }^{35}$ Moreover, recently Volpe et al. ${ }^{36}$ and Dregely et al. ${ }^{37}$ have used PMMA and HSQ respectively to map the optical near-field of plasmonic Au nanoantennas. Volpe et al. observed that PMMA decomposed within the plasmonic hotspots of Au nanorods illuminated at a resonant wavelength of $800 \mathrm{~nm}$. They also found that the volume of decomposed PMMA scaled with the fourth power of the laser intensity, which suggests an exposure mechanism based on 4-photon absorption. Jiang and Gordon ${ }^{38}$ later showed that the mechanism proposed by Volpe et al. may not produce sufficient PMMA decomposition to support lithographic activity. Additionally, neither the work of Volpe et al., nor that of Jiang and Gordon, considered an exposure mechanism based on electron emission from the plasmonic nanoparticles. However, as we know from previous work,,$^{20,25,26,39-41}$ femtosecond lasers can drive significant electron emission currents from plasmonic nanoparticles and produce extremely high peak current densities in such particles. 
In this work, we show that the electron emission from Au nanoantennas excited by femtosecond laser pulses is sufficient to expose both PMMA and HSQ. Moreover, we show that the spatial distribution of the exposed PMMA is in agreement with the expected distribution of emitted electrons based on simulations of both the optical near-field and the trajectories of electrons emitted by strong-field tunneling emission. In contrast to Volpe et al., we have used a laser source with a central wavelength of $1200 \mathrm{~nm}$ to excite the LSPRs in our Au nanorods. As such, cumulative absorption of 6 photons would be required to expose PMMA by multiphoton absorption (given that PMMA absorbs weakly at wavelengths longer than $200 \mathrm{~nm}$ ); we exploit the reduced probability of 6-photon absorption to enable nanometer mapping of electron emission in PMMA. As stated earlier, we also used HSQ as an imaging layer to map electron emission and energy transfer from plasmonic nanoantennas. Similar to PMMA, exposed HSQ was also observed at the poles of nanorod antennas when it was used as an imaging layer. The observed exposure at the poles of nanorods is in agreement with the results of experiments using PMMA and likewise supports an exposure mechanism based on field-driven electron emission from plasmonic hot spots. In contrast to the PMMA results, exposed HSQ was additionally observed at the center of nanorod antennas where surface fields are expected to be lowest. We propose that exposure of HSQ in this region is due to the local generation of hot electrons locally at the center of the nanorods where conduction current densities and consequently, resistive, i.e. Ohmic, losses in the nanorods are at a maximum. ${ }^{33,42,43}$ Jermyn et al. for example, have recently reported the results of models for the energetic and spatial distributions of hot electrons in Au nanorods that are in excellent agreement with HSQ exposures observed in this work. ${ }^{43}$ In this work, we coarsely estimate the energy of hot electrons generated in the nanoantennas by calculating resistive losses and assuming that on the timescale of the laser pulse all power dissipated by resistive loss is carried 
by conduction electrons in Au. Based on these assumptions and approximations, we hypothesize that hot electrons $1.5 \mathrm{eV}$ above the Fermi level of Au may have sufficient energy to initiate the dissociation of water, which subsequently drives hydrolysis and crosslinking of HSQ. Our investigations using HSQ as an imaging layer suggest that we may spatially distinguish between electrons emitted due to field-dependent photoemission at nanorod poles and hot electrons generated at the center of the nanorods. As such, this work shows that lithographic materials may provide a number of opportunities to study the roles of plasmons in energy transfer at the nanometer length scale and femtosecond timescale.

Figure 1 outlines the experimental approach used in this work. Arrays of Au nanoantennas such as those shown in figures 1a,b were fabricated on indium-tin-oxide-coated sapphire substrates by electron-beam lithography (see supporting information and previous reports ${ }^{20,25}$ for details). The nanoantenna arrays were prepared without a conventional adhesion promoting layer such as $\mathrm{Ti}$ or $\mathrm{Cr}$ as these layers have been shown to detrimentally affect optical field enhancement. ${ }^{20,44}$ Photoemission from the nanoantenna arrays was measured using the setup shown schematically in figure $1 \mathrm{c}$ (the setup is further described in our recent report $\mathrm{t}^{25}$ ). The nanoantennas fabricated in this work typically produced $\sim 1$ electron per nanoantenna per laser pulse or equivalently, $1-10 \mathrm{C} / \mathrm{cm}^{2}$ $\left(10^{4}-10^{5}\right.$ electrons $\left./ \mathrm{nm}^{2}\right)$ at the poles of the nanoantennas (see supporting information). We coated the nanoantenna arrays with a thin film of electron-beam resist: polymethyl(methacrylate) (PMMA) or hydrogen silsesquioxane (HSQ). The resist was exposed by illuminating the nanoantennas with the femtosecond laser source $(12.5 \mathrm{~mW}$ average power, $\approx 10 \mathrm{fs}$ pulse, 78.4 $\mathrm{MHz}$ repetition rate, $10 \mathrm{~s}$ exposure time, $\sim 0.6 \mathrm{MJcm}^{-2}$ average fluence per exposure). Following 
exposure, we removed the exposed (PMMA) or unexposed (HSQ) resist using an appropriate developer (see supporting information).
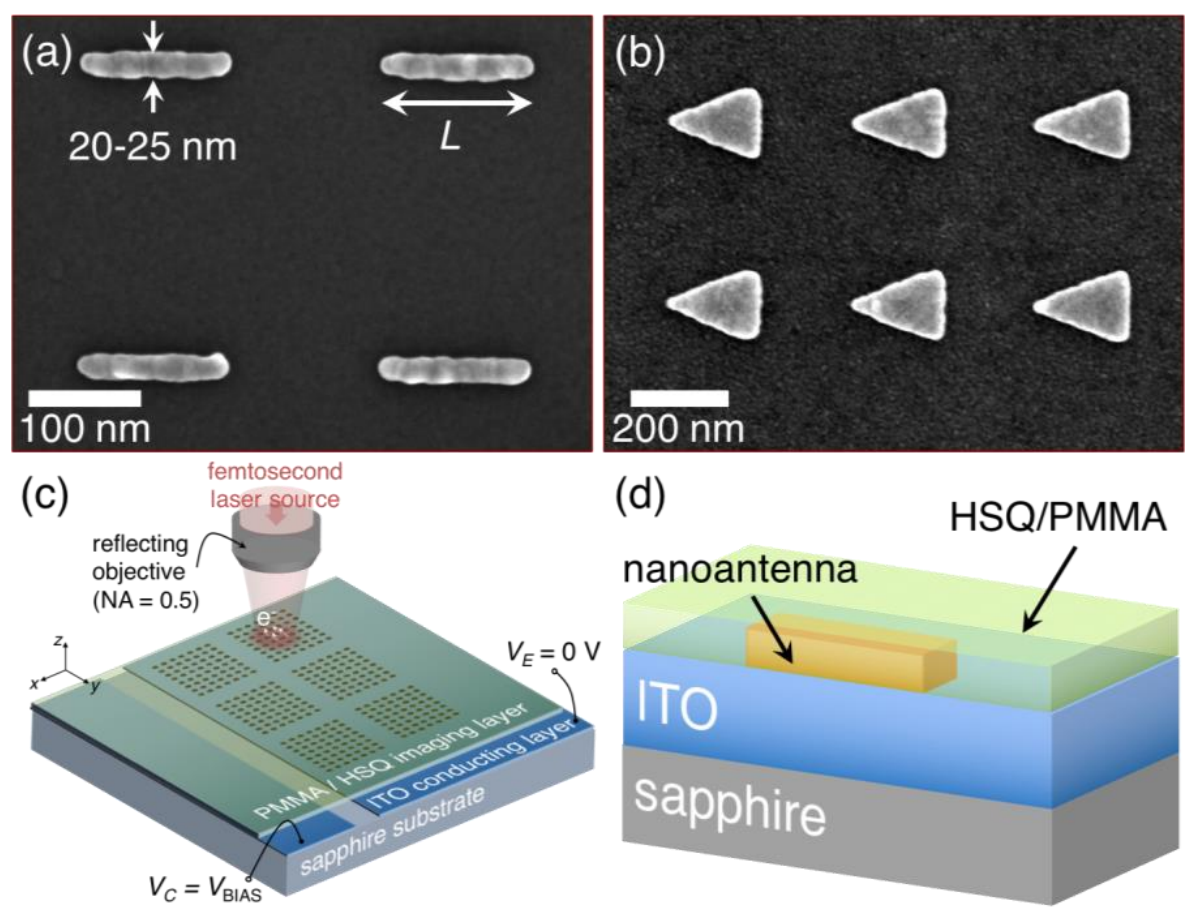

(d)

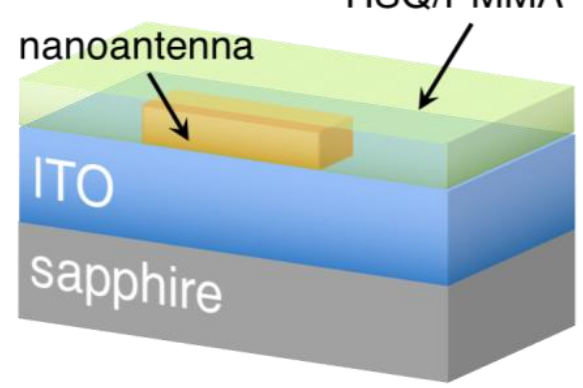

Figure 1. Nanoantennas and experimental approach. (a,b) Top-down SEM micrographs of an array of $\mathrm{Au}$ nanorod (a) and nanotriangle (b) antennas fabricated by electron-beam lithography. (c) Schematic representation of experimental setup. 10 fs pulses of linearly polarized light with a central wavelength of $1.2 \mu \mathrm{m}$ and a bandwidth of $400 \mathrm{~nm}$ were focused to a $1 / \mathrm{e}^{2}$ diameter of $5.2 \mu \mathrm{m}$ on the nanoantenna arrays, which were fabricated on an ITO-coated (blue regions) sapphire substrate (grey regions). A $5 \mu \mathrm{m}$ gap was etched in the ITO layer as shown to allow a bias to be applied between the nanoantenna emitter array (the emitter electrode: blue region on the right; $\mathrm{V}_{\mathrm{E}}=0$ ) and the collector electrode (blue region on the left; $\mathrm{V}_{\mathrm{C}}=\mathrm{V}_{\mathrm{BIAS}}$ ). (d) Schematic showing a nanoantenna coated with a 20-nm-thick layer of PMMA or HSQ (semitransparent green region), which acted as an imaging layer for emitted electrons. 
Figure 2 shows SEM images of both nanorod and nanotriangle antennas that were coated with 20-25 nm of PMMA, exposed with the femtosecond laser source and developed to remove the exposed PMMA. We used a low-temperature development process to improve the contrast of the features produced in PMMA (see supporting information for details). Figure 2a shows a lowmagnification SEM image of the exposed area of a 260-nm-pitch square array of 130-nm-long Au nanorod antennas after developing the exposed PMMA. The nanorod arrays were exposed to $\sim 8$ $\times 10^{8}$ laser pulses each having an energy of $0.16 \mathrm{~nJ}$. Figure $2 \mathrm{~b}$ shows an SEM image of a nanorod near the periphery of the exposed area shown in figure $2 \mathrm{a}$. The nanorod shows well-defined regions of exposed PMMA at its poles where the optical fields peaked and thus where electron emission is expected to be most efficient.

Figures $2 \mathrm{a}$ and $2 \mathrm{~b}$ show that PMMA is exposed at distances of more than $50 \mathrm{~nm}$ from the poles of the Au nanoantennas. This is a rather surprising result considering that the emitted electrons are expected to have kinetic energies of $<10 \mathrm{eV}$ and intuitively might be expected to propagate shorter distances $(<50 \mathrm{~nm})$ in PMMA based on universal inelastic mean-free path (IMFP) curves. $^{45}$ However, low-energy electrons have been observed to expose resists at distances of $\sim 100 \mathrm{~nm}$ from their point of origin; for example, Duan et al. ${ }^{46}$ measured the point-spread function of a $30 \mathrm{keV}$ electron beam in HSQ on a 50-nm-thick freestanding membrane and observed resist exposure $100 \mathrm{~nm}$ from the point of exposure. Their result suggests that low-energy secondary electrons are capable of exposing resist at significant distances from the point of exposure when the dose is sufficiently high. Moreover, while the IMFP of electrons typically decreases with decreasing electron energy, reports suggest that the IMFP rises steeply when the electron energy drops below $10 \mathrm{eV} \cdot{ }^{45,47,48}$ 
An important property of PMMA is that it is a dual-tone electron-beam resist; PMMA behaves as a positive-tone resist at low doses and as a negative-tone resist when exposed to high doses of electrons. ${ }^{49}$ Briefly, when exposed to low doses of electrons, e.g. $\sim 10$ electron $/ \mathrm{nm}^{2}$ for $30 \mathrm{keV}$ electrons, the long polymer chains in PMMA decompose into smaller, lower molecular weight chains that may be readily removed by an appropriate solvent (the developer). However, when PMMA is exposed to higher electron doses, e.g. $\sim 10^{4}$ electron $/ \mathrm{nm}^{2}$ for $30 \mathrm{keV}$ electrons, the lowmolecular-weight fragments of PMMA can cross-link to form higher molecular weight species (typically referred to as negative-tone PMMA in the field of electron-beam lithography). ${ }^{49} \mathrm{~A}$ local incident laser intensity of $\sim 0.1 \mathrm{GW} / \mathrm{cm}^{2}$ was required to observe positive-tone exposure of PMMA in the hot spots of nanoantennas in this work.

Figure 2c shows an SEM image of a nanorod near the center of the exposed region displayed in figure 2a. A narrow strip of material resembling crosslinked negative-tone PMMA is present at the apex of the nanorod shown. This negative-tone PMMA indicates that this location was where the PMMA received the highest electron-exposure dose. A local incident laser intensity of $\sim 50$ $\mathrm{GW} / \mathrm{cm}^{2}$ was required to observe negative-tone exposure of PMMA for nanoantennas excited along their long axis in this work. Additionally, in figures $2 \mathrm{~d}$ and $2 \mathrm{e}$, we show the polarization dependence of the PMMA exposure for Au nanotriangle antennas. Figure 2d shows an SEM image of a Au nanotriangle exposed with the linear polarization of our source aligned with the long-axis of the triangle; the resulting PMMA exposure appears at the apex of the long-axis. Figure 2e shows an SEM image of a nanotriangle exposed with orthogonal polarization; here, the exposed PMMA appears at the other apices of the triangle. Negative-tone exposure of PMMA was not observed for nanotriangles excited by light having linear polarization aligned to the short axis of triangles. 

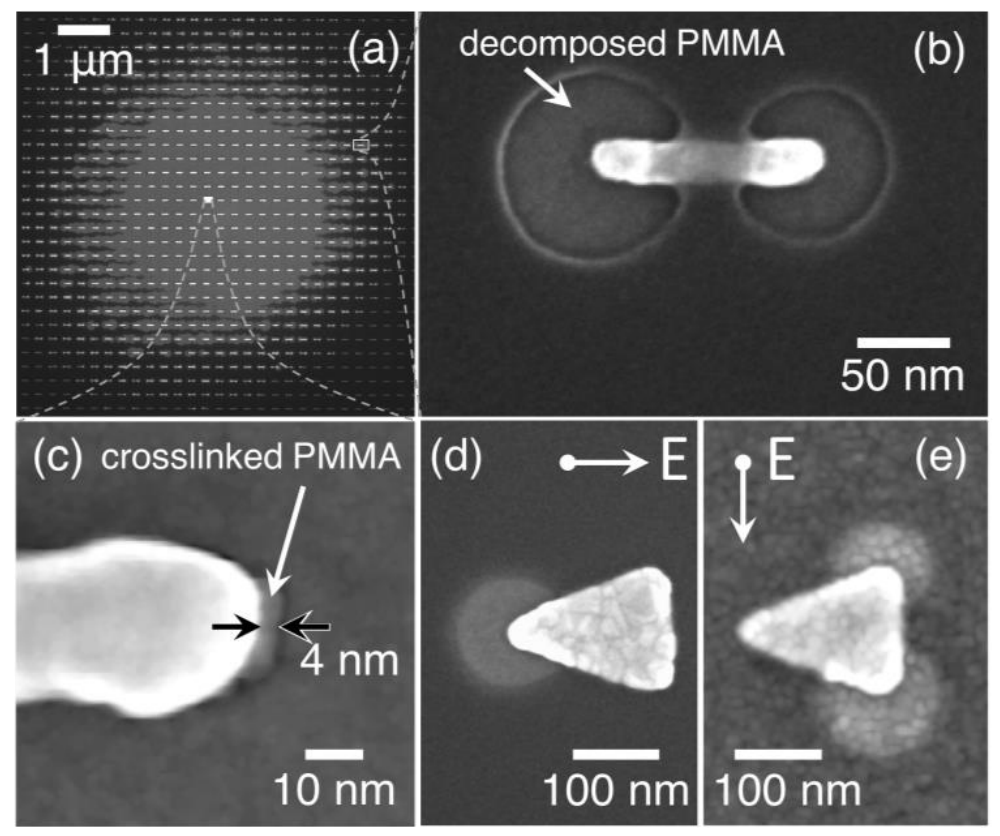

Figure 2. Imaging electron emission from plasmonic nanoantennas with PMMA. (a) Top-down SEM micrograph of a 260-nm-pitch Au nanorod array coated with a 20-nm-thick layer of PMMA. The coated array was previously exposed to $\sim 10^{9}$ femtosecond laser pulses, and the PMMA was subsequently developed. (b) SEM micrograph of a nanorod antenna near the periphery of the exposed region shown in (a). The image shows regions of exposed and developed PMMA at the poles of the nanoantenna. (c) SEM micrograph of a pole of a nanorod near the center of the exposed region in (a). A narrow strip of material believed to be cross-linked PMMA is present at the apex of the nanorod. $(\mathrm{d}, \mathrm{e})$ SEM micrographs of exposed PMMA around nanotriangles illuminated with $\sim 10^{9}$ femtosecond laser pulses. The polarization of these pulses was aligned (d) parallel to and (e) orthogonal to the long-axis of the nanotriangle antennas.

To better understand the observed PMMA exposure and correlate the distribution of exposed PMMA to simulations of the local optical-field enhancement and photoelectron distribution, we investigated the dependence of PMMA exposure on the LSPR of Au nanorods. The results of these 
investigations are summarized in figure 3. Figure 3a shows (black data points) the volume $(V)$ of exposed and developed PMMA at the poles of Au nanorods 2.5-3.0 $\mu \mathrm{m}$ from the center of the exposed spot (estimated by inspection of SEM images; see details in supporting information). We measured $V$ for 5-10 poles for each nanorod length $(L)$ and plotted these values against $L$. The error bars on the values of $V$ represent the maximum and minimum values measured in the region 2.5-3.0 $\mu \mathrm{m}$ from the center of the laser spot. The plot also shows the spectrum of the femtosecond laser source (red trace). We aligned the upper and lower $x$-axes of the plots in figure $3 \mathrm{a}$ by simulating the extinction spectra of nanorods having the lengths shown and mapping the value of nanorod length to the corresponding wavelength of the simulated LSPR peak. The plot in figure 3a shows that PMMA exposure was only observed for nanorods having a LSPR overlapping the laser spectrum.

Figure $3 \mathrm{~b}$ shows an example SEM image of a Au nanorod inspected for use in the preparation of the plot shown in figure 3a. Regions of exposed and developed PMMA are clearly seen at the poles of the nanorod antenna. Figure 3c shows an SEM image of a 130-nm-long, 20-nm-wide Au nanorod antenna, and a color map of the simulated optical field-enhancement is overlaid in the upper left quadrant of the image. The regions of exposed PMMA produced by our experiment and shown in the SEM image in figure $3 b$ overlap well with the regions displaying strong field enhancement (shown in figure 3c). The results of the simulated optical near-field were used to estimate optical-field-driven photoemission currents from these plasmonic nanoantennas using a Fowler-Nordheim model for electron emission. ${ }^{20,50,51}$ The trajectories of emitted electrons were additionally simulated using a particle-in-cell model to produce a map of the distribution of electrons colliding with the ITO substrate after emission from the plasmonic antenna. ${ }^{51}$ Simulations of electron trajectories were performed in vacuum without the overlying PMMA layer 
for simplicity; however, simulations of the antenna near-field did include the PMMA layer to best represent the local field profile. A color map of the number of emitted electrons recombining with the substrate is overlaid in the lower left quadrant of the SEM image in figure 3c (further details are provided in the supporting information and in a previous report ${ }^{51}$ ). The electron-emission simulations suggest that $10^{4}-10^{5}$ electrons $/ \mathrm{nm}^{2}$ were incident on the substrate near the apex of the nanorod. This estimate is in good agreement with the measured emission currents of $10^{4}-10^{5}$ electrons $/ \mathrm{nm}^{2}$ (see supporting information). Both the simulated and measured electron doses calculated at the poles of the plasmonic nanoantennas are commensurate with the doses required to expose PMMA with electrons in the 1-50 eV energy range. For example, McCord and Pease patterned PMMA using a scanning probe lithography technique with $20 \mathrm{eV}$ electrons and observed positive-tone behavior in PMMA at doses of $10^{3}-10^{5}$ electron $/ \mathrm{nm}^{2} .52$ 

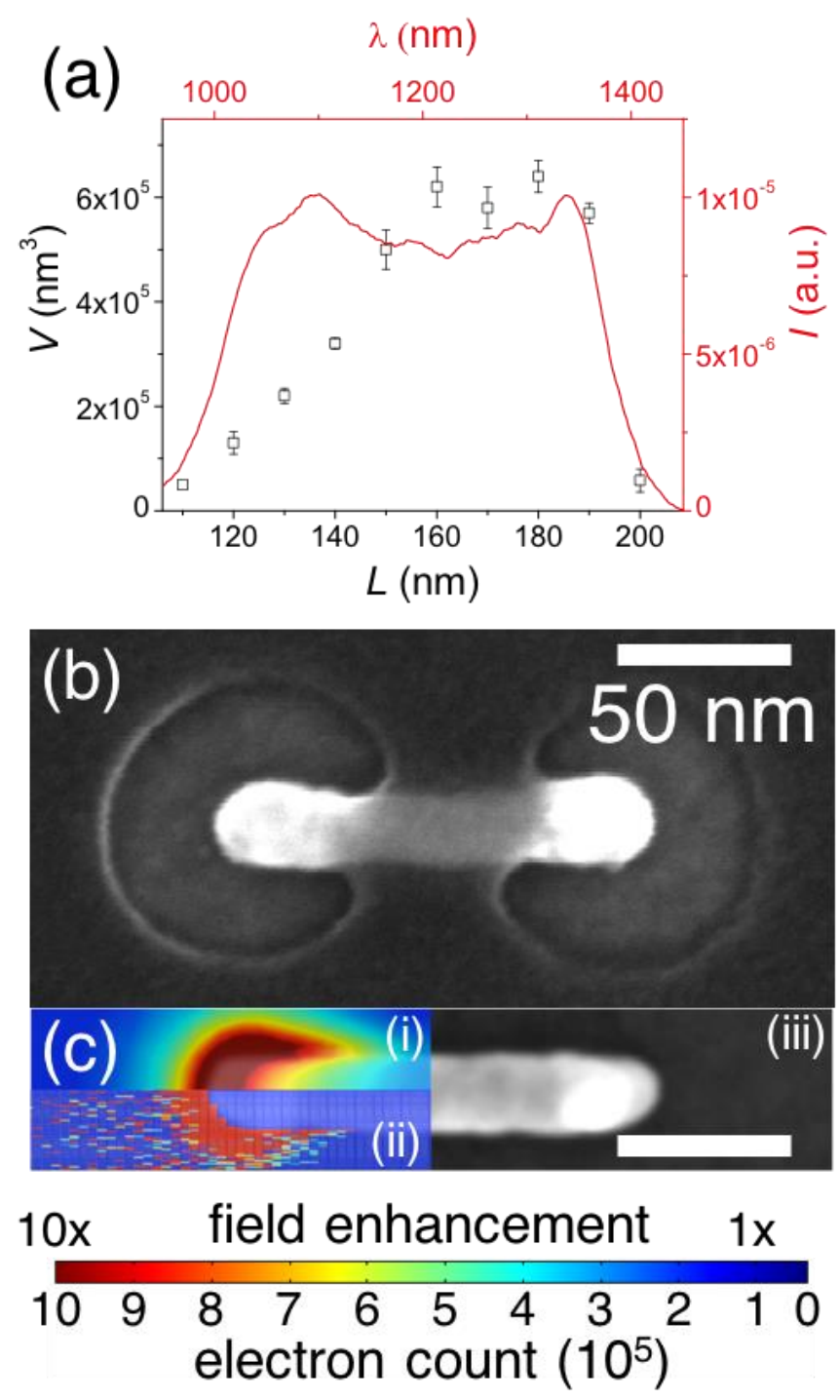

Figure 3. PMMA exposure versus the nanorod resonance, field-enhancement at the nanorods, and spatial distribution of emitted photoelectrons. (a) Black data represent a plot of the mean volume $(V)$ of developed PMMA at the pole of a nanorod antenna of length $(L)$; the volume was measured for nanorods a distance of $\approx 2.5-3.0 \mu \mathrm{m}$ from the center of the laser spot. Error bars represent the maximum and minimum values of $V$ for each nanorod length. The red curve represents the normalized intensity $(I)$ spectrum of the laser used to excite electron emission. (Note that the pitch of each nanorod array was $4 L$.) (b) Example of an SEM micrograph used to measure the volume of developed PMMA. (c) (i) A simulated map of the magnitude of optical field enhancement at 
the pole of 130-nm-long nanorod antenna (scale is saturated at a field-enhancement factor of 10, while the field-enhancement peaks at 25 near the nanorod apex). (ii) A map of the simulated number of electrons passing through a plane at the air-ITO interface. (iii) SEM micrograph of a 130-nm-long Au nanorod (scale bar $50 \mathrm{~nm}$ ).

In this work, we also used thin films of HSQ as imaging layers for electron emission and energy transfer from plasmonic nanorods. HSQ is an inorganic negative-tone electron-beam resist that crosslinks under electron exposure. The mechanism of electron-beam-induced crosslinking of HSQ is reported to involve the hydrolysis of the silane groups in the presence of water derived from ambient vapor and a subsequent condensation reaction forming siloxane bonds resulting in the formation of a silicon oxide network that is insoluble in HSQ developer solutions. ${ }^{53}$

Figure 4 displays a summary of the results observed when using HSQ as an imaging layer for electron emission from plasmonic $\mathrm{Au}$ nanorod antenna arrays. Unlike PMMA, where resist exposure was primarily observed at the poles of nanoantennas, crosslinked HSQ was observed at both the centers and the poles of nanorods as shown in the SEM images in figures $4 \mathrm{a}$ and $4 \mathrm{~b}$. As discussed, optical-field-driven electron emission can explain HSQ and PMMA exposure at the nanorod poles (this is where the field-enhancement is at a maximum). However, the enhancement of surface fields is at a minimum at the center of nanorods having lengths in the range studied here. Consequently, another mechanism must be invoked to describe the energy-transfer process that results in the observed exposure at the center of nanorod antennas.

The transfer of energy from plasmonic nanoparticles to semiconductors and surface-bound molecules is an area that has received significant attention in recent years. The mechanisms of energy transfer have been summarized in a number of recent reports. ${ }^{4,29,54-58}$ These mechanisms 
can be categorized into four major sub-types: (1) plasmon-induced hot-electron transfer, (2) plasmon-induced direct electron transfer (PIDET), (3) plasmon-induced resonant energy transfer (PIRET) and (4) plasmon-induced radiative energy transfer. In the first sub-type, hot electrons are generated in the plasmonic nanoparticle and are subsequently injected into accessible states in the adsorbate. The PIDET process, which is often also referred to as chemical-interface damping, involves the direct transfer of an electron from the metal to the surface-bound material. The transition energy for the PIDET process should overlap with the LSPR energy. The PIRET process can be described by energy transfer via resonant coupling between the dipole of the plasmonic antenna and the dipole of an electron-hole pair in the adsorbate. The last transfer mechanism involves radiative decay of the plasmon and absorption of the emitted photon in the adsorbate. In this work, we expect that photons produced by radiative decay will have insufficient energy to expose PMMA or HSQ. Similarly, the PIRET process is not expected to enable transitions having sufficient energy to expose resists. A PIDET process could excite electrons in Au to levels 0.9-1.2 eV (range of LSPRs that may be excited with our laser source) above the Fermi level, which may be sufficient to dissociate surface-bound species. However, it is expected that the PIDET process would be favored at the poles of antennas based on the electric-field dependence of transition probabilities described by Fermi's golden rule. ${ }^{56}$ In this work, we posit that hot electrons generated by plasmon decay are responsible for the exposure of HSQ at the center of nanorods and we further speculate as to a possible exposure mechanism based on hot-electron transfer to water leading to hydrolysis of HSQ. The mechanism presented is entirely speculative at this point and further work is required to confirm the exact mechanism. 
The current density due to conduction electrons involved in the LSPR of plasmonic nanorod antennas peaks at the center of the nanorods. ${ }^{33,42}$ Consequently, power dissipation due to resistive losses is also strongest at the center of nanorods. On the timescale of the femtosecond laser pulses used in this work, power dissipated by the LSPR is expected to be carried primarily by the conduction electrons involved in the LSPR. As such, power dissipated by the LSPR is likely to generate a non-equilibrium distribution of hot electrons near the center of the nanorods. The recent report by Jermyn et al. shows that the distribution of hot carriers is expected to peak at the center of nanorod antennas. ${ }^{43}$ These hot electrons may carry sufficient energy to dissociate species on the Au surface via mechanisms such as dissociative electron attachment. ${ }^{59,60}$ Dissociation of water due to hot electrons could play an important role in the crosslinking of HSQ, as water dissociation products would support the hydrolysis of silane groups in HSQ. In this work, all experiments were performed under ambient conditions in the presence of airborne water vapor and thus surfacebound water is expected. Notably, the lowest unoccupied molecular orbital (LUMO) of water adsorbed on Au surfaces is expected to be $1-2 \mathrm{eV}$ above the Au Fermi level, consequently we hypothesize that hot electrons having energies in this range may support crosslinking of HSQ. ${ }^{61-}$ 64

Here, we approximate the power dissipated locally across nanoantennas by calculating the resistive loss for nanorod antennas under continuous-wave illumination (specifically, we calculate the average Ohmic losses in the nanoantennas i.e. $1 / 2 \operatorname{Re}\left\{\boldsymbol{J} \cdot \boldsymbol{E}^{*}\right\}$ where $\boldsymbol{J}$ is the induced current in the nanoantenna and $\boldsymbol{E}$ is the local electric field). We then make the assumption that the dissipated power is carried by conduction electrons in Au and we calculate the average energy deposited per conduction electron at the center of nanorods to coarsely determine the energy of hot electrons. 
Figure 4c shows a color map of the simulated local resistive loss across a 210-nm-long nanorod overlaid on a SEM image of an exposed HSQ-coated nanorod of the same length.

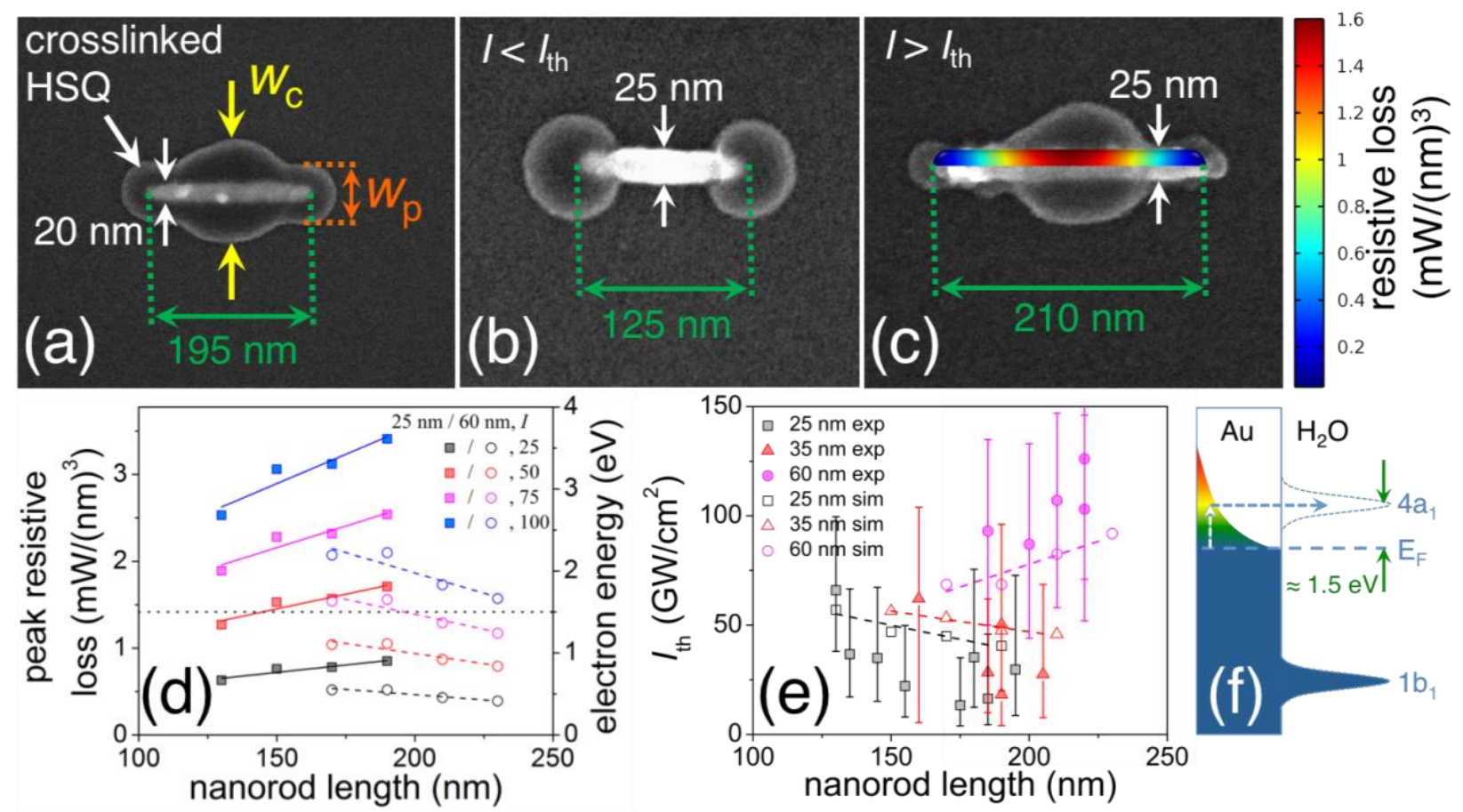

Figure 4. Mapping electron emission in a HSQ imaging layer. (a) Cross-linked HSQ was observed at the poles of nanorod antennas and the centers of antennas as shown in the SEM image. At a threshold laser intensity $I_{\mathrm{th}}$, the width of crosslinked HSQ at the antenna center $\left(w_{\mathrm{c}}\right)$ is greater than that at the poles $\left(w_{\mathrm{p}}\right)$. (b) Example SEM image of a nanoantenna exposed at an intensity $I<I_{\text {th }}$ where there was greater crosslinking at the poles of the antennas as shown. (c) Dominant HSQ exposure at the center of nanoantennas $\left(w_{\mathrm{c}}>w_{\mathrm{p}}\right)$ was found to vary with nanorod length and width as shown. The SEM image in (c) shows a 25-nm-wide, 210-nm-long Au nanorod having $w_{\mathrm{c}}>w_{\mathrm{p}}$. A color map of the simulated local resistive losses across the nanorod is overlaid on the SEM image; resistive losses were calculated to be strongest at the center of the nanorod. (d) Plot of simulated peak resistive loss at the center of Au nanoantennas (left axis) and calculated electron energy (right axis) against nanorod length. Filled squares represent 25-nm- 
wide nanorods, and open circles represent 60 -nm-wide nanorods. The laser intensity $I$ used in the simulations is given in the legend in units of $\mathrm{GW} / \mathrm{cm}^{2}$. The dashed horizontal line represents a peak resistive loss of $1.42 \mathrm{~mW} / \mathrm{nm}^{3}$ or hot-electron energy of $1.5 \mathrm{eV}$. (e) Ith required to generate dominant exposure of HSQ at the center of the nanorods $\left(w_{\mathrm{c}}>w_{\mathrm{p}}\right)$ plotted against nanorod length. Filled data points represent measured values for nanorods having widths as specified in the legend. Open data points represent simulated $I_{\text {th }}$ values required to produce hot electrons 1.5 $\mathrm{eV}$ above the Fermi level. Error bars represent the intensity range covered by a unit cell of the nanoantenna array (unit cell measures $4 L \times 4 L$ ). (f) Speculated mechanism of hot-electroninduced decomposition of water on an Au surface. A hot electron with sufficient energy (dashed white arrow) may be excited from the Fermi level of gold $\left(E_{\mathrm{F}}\right)$ and injected into an anti-bonding orbital of water (4a1) resulting in ionization and dissociation of water followed by crosslinking of HSQ.

To investigate the role of resistive loss in HSQ exposure, we first compared the relative exposure of HSQ at the center of nanorods to that at the poles for nanorods having different lengths and widths. We used the relative widths of crosslinked HSQ at the center $\left(w_{\mathrm{c}}\right)$ and poles $\left(w_{\mathrm{p}}\right)$ of nanorods (see figure 4a) as a metric to study the mechanism of HSQ exposure at the center of nanorod antennas. We classified exposed nanorods into two distinct types: nanorods with dominant HSQ exposure at their centers $\left(w_{\mathrm{c}}>w_{\mathrm{p}}\right)$, such as the one shown in figures $4 \mathrm{a}$, and nanorods with dominant exposure at their poles $\left(w_{\mathrm{c}}<w_{\mathrm{p}}\right)$, like the one shown in figure $4 \mathrm{~b}$. We observed $w_{\mathrm{c}}>w_{\mathrm{p}}$ at the center of the laser spot, that is, in regions exposed to the highest laser intensities, and we determined a threshold intensity $I_{\text {th }}$ that is required to produce HSQ exposure where $w_{\mathrm{c}}>w_{\mathrm{p}}$.

To better understand the relationship between resistive power loss, laser intensity and nanorod geometry, we simulated resistive loss in nanorods having different geometries and illuminated 
with varying laser intensity. Figure $4 \mathrm{~d}$ shows a plot of peak resistive loss (left axis) and hot electron energy (right axis) against nanorod length; the simulated nanorods were illuminated at their resonant wavelength and had two different widths $(25 \mathrm{~nm}$ and $60 \mathrm{~nm})$. Here, peak resistive loss refers to the maximum simulated resistive loss in a nanorod for a given illumination intensity. The peak resistive loss was always observed at the surface of the nanorods midway along their length as seen in figure 4c. We converted values of peak resistive loss to estimates of hot-electron energy using the following simplified approach: we assumed that all of the power deposited due to resistive loss was transferred on the timescale of the femtosecond laser pulse (10 fs) to free electrons in $\mathrm{Au}$ (present with a density of 59 electron $/ \mathrm{nm}^{3}$ ); we thus obtained an average energy deposited per free electron. ${ }^{65}$ We observed that peak resistive loss increased with laser intensity for both rod widths studied. However, while resistive loss increased with rod length for 25-nmwide nanorods it decreased with increasing rod length for 60 -nm-wide nanorods, for the range or lengths studied. Figure $4 \mathrm{~d}$ shows that, for any given laser intensity, 60-nm-wide nanorods are expected to exhibit lower resistive loss than 25 -nm-wide rods. The results shown in figure $4 \mathrm{~d}$ suggest that wider nanorods will require higher values of threshold laser intensity $I_{\text {th }}$ to produce dominant HSQ exposure at their center and that $I_{\text {th }}$ should increase with $L$ for 60 -nm-wide nanorods, while showing the opposite trend for the narrower rods over the range of lengths studied. This difference in behavior of 60-nm-wide nanorods compared to 25-nm-wide nanorods can largely be explained by the increased radiative plasmon decay (scattering) relative to non-radiative decay (absorption) expected for the wider rods. Figure S9 in the supporting information provides plots of simulated absorption and scattering cross-sections for various nanorod geometries and highlights the increased contribution of scattering for 60 -nm-wide nanorods with increasing rod length. 
In figure 4e, we plot the measured threshold intensity (filled data points) against $L$ for nanorods having widths of $25 \mathrm{~nm}, 35 \mathrm{~nm}$ and $60 \mathrm{~nm}$. Details of the method used to extrapolate values of $I_{\text {th }}$ and the associated error bars are provided in the supporting information. The range covered by the error bars is representative of the local intensity in the Gaussian laser spot $\pm 2 L$ (half a unit cell in the nanoantenna array) from the point where the value of $I_{\text {th }}$ was measured. In figure $4 \mathrm{e}$ we can see that the measured values of $I_{\text {th }}$ decrease with increasing $L$ for 25 -nm-wide and 35 -nm-wide rods, while $I_{\text {th }}$ increases with $L$ for 60 -nm-wide rods. Moreover, we can see that the values of $I_{\text {th }}$ for the 60-nm-wide nanorods are significantly higher than those for narrower rods. All of the above trends are in agreement with the trends predicted by figure $4 \mathrm{~d}$. Figure $4 \mathrm{e}$ also shows the simulated values of $I_{\text {th }}$ required to produce peak resistive losses of $1.42 \mathrm{~mW} / \mathrm{nm}^{3}$ or equivalently, hot electrons having energies of $1.5 \mathrm{eV}$ (open data points). The simulated values reside within the error bars of the measured values and support the speculated HSQ-exposure mechanism based on hotelectron production via resistive losses.

As stated previously, water-mediated hydrolysis of HSQ is thought to play a major role in the crosslinking of HSQ. Dissociative electron attachment has been observed to produce hydride anions via resonant ionization in thin films of water ${ }^{64}$ The resonance with the largest cross-section in water is the ${ }^{2} \mathrm{~B}_{1}$ resonance and involves the generation of a hole in the $1 \mathrm{~b}_{1}$ level associated with a lone pair on the oxygen atom and electronic occupation of the $4 \mathrm{a}_{1}$ anti-bonding orbital. ${ }^{64}$ The energy of the ${ }^{2} \mathrm{~B}_{1}$ resonance has been reported as $6.5 \mathrm{eV}$, which means that the $4 \mathrm{a}_{1}$ anti-bonding orbital is approximately $1.5 \mathrm{eV}$ above the Fermi level of ice (figure $4 \mathrm{f}$ ) given that the $1 \mathrm{~b}_{1}$ level has been measured at $5 \mathrm{eV}$ below the Fermi level. Consequently, we hypothesize that hot electrons produced at the center of the Au nanorods may be injected into anti-bonding orbitals of water and that could then drive ionization by dissociative electron attachment or similar mechanisms. ${ }^{66}$ The 
resultant water decomposition products would then support crosslinking of HSQ, which initiates at the center of the nanorod. Exposure of HSQ $\sim 10 \mathrm{~nm}$ from the surface of nanoantennas could be explained by the diffusion of water decomposition products through the porous exposed HSQ closer to the surface. Acid diffusion lengths in this range have been observed in studies of chemically-amplified resists, for example. ${ }^{67}$ Further work is required to confirm the proposed HSQ exposure mechanism, but the spatial localization of crosslinked HSQ at the center of the nanorods and the observed dependence of this crosslinked HSQ on nanorod length and width suggest that resistive loss at the center of nanorods is responsible for HSQ exposure in this region.

\section{Conclusions}

In this work, we have shown that electron-beam resists can be used to map electron emission from plasmonic nanoantennas with nanometer-scale resolution. The doses required to expose PMMA via electrons emission from plasmonic antennas are consistent with those previously measured in low-electron-energy scanning-probe lithography. Our simulations of the spatial distribution of the optical near-field and emitted electrons are in good agreement with the observed features of the exposed PMMA. Additionally, we observed exposure of HSQ at both the poles and centers of the nanorod antennas and we have correlated HSQ exposure at the center of the nanorods to resistive losses in the rods. Specifically, we have seen that the dependence of the magnitude of the HSQ exposure at the center of the nanorods on the dimensions of the rods is in good agreement with the dependence displayed by resistive losses in the nanoantennas. These results suggest the possibility of controlling hot electron distributions via nanostructure geometry and such control presents an opportunity to engineer plasmonic nanoantennas tailored for specific photochemical applications by controlling the location and energy of hot electrons transferred from metallic 
plasmonic nanoantennas to molecular species at their surface. Moreover, as has been highlighted recently, ${ }^{68}$ the ability to controllably pattern the surfaces of nanoparticles is desirable for applications in sensing and catalysis. As a result, the method outlined in this work may also provide a route to the production of nanoparticles with surface patterns that can be controlled the methods described here and used for the development of new photocatalyst and optoelectronic systems.

\section{Acknowledgements}

RGH, YY, and KKB would like to also acknowledge support from the Gordon and Betty Moore Foundation for support of simulations of energy deposition. RGH was supported for work in fabrication of structures, metrology and data analysis by the Center for Excitonics, an Energy Frontier Research Center funded by the U.S. Department of Energy, Office of Science, Office of Basic Energy Sciences, under Award Number DE-SC0001088. RGH also acknowledges support from the Royal Society-Science Foundation Ireland University Research Fellowship. We thank James Daley and Mark Mondol for assistance with nanofabrication of gold nanoantennas.

\section{Notes}

The authors declare no competing financial interests.

\section{Supporting Information}

Further details related to fabrication of nanorods, lithographic processing, analysis of SEM micrographs, estimation of electron emission yields and electromagnetic simulations are included in the supporting information. This information is available free of charge via the Internet at http://pubs.acs.org. 


\section{References}

(1) Lal, S.; Link, S.; Halas, N. J. Nat. Photonics 2007, 1, 641-648.

(2) Atwater, H. A. Sci. Am. 2007, 296, 56.

(3) Maier, S. A.; Brongersma, M. L.; Kik, P. G.; Meltzer, S.; Requicha, A. A. G.; Atwater, H. A. Adv. Mater. 2001, 13 (19), 1501-1505.

(4) Brongersma, M. L.; Halas, N. J.; Nordlander, P. Nat. Nanotechnol. 2015, 10, 25-34.

(5) Cortés, E.; Xie, W.; Cambiasso, J.; Jermyn, A. S.; Sundararaman, R.; Narang, P.; Schlucker, S.; Maier, S. A. Nat. Commun. 2017, 8, 14880.

(6) Anker, J. N.; Hall, W. P.; Lyandres, O.; Shah, N. C.; Zhao, J.; Duyne, R. P. Van. Nat. Mater. 2008, 7, 442-453.

(7) Mallidi, S.; Larson, T.; Tam, J.; Joshi, P. P.; Karpiouk, A.; Sokolov, K.; Emelianov, S. Nano Lett. 2009, 9, 2825-2831.

(8) Steuwe, C.; Erdelyi, M.; Szekeres, G.; Csete, M.; Baumberg, J. J.; Mahajan, S.; Kaminski, C. F. Nano Lett. 2015, 15, 3217-3223.

(9) Dvořák, P.; Neuman, T.; Bř́nek, L.; Šamořil, T.; Kalousek, R.; Dub, P.; Varga, P.; Šikola, T. Nano Lett. 2013, 13, 2558-2563.

(10) Atwater, H. A.; Polman, A. Nat. Mater. 2010, 9, 865-865.

(11) Sheldon, M. T.; van de Groep, J.; Brown, A. M.; Polman, A.; Atwater, H. A. Science 2014, $346,828-831$. 
(12) Awazu, K.; Fujimaki, M.; Rockstuhl, C.; Tominaga, J.; Murakami, H.; Ohki, Y.; Yoshida, N.; Watanabe, T. J. Am. Chem. Soc. 2008, 130, 1676-1680.

(13) Christopher, P.; Xin, H.; Linic, S. Nat. Chem. 2011, 3, 467-472.

(14) Di Martino, G.; Michaelis, F. B.; Salmon, A. R.; Hofmann, S.; Baumberg, J. J. Nano Lett. 2015, $15,7452-7457$.

(15) Minamimoto, H.; Toda, T.; Futashima, R.; Li, X.; Suzuki, K.; Yasuda, S.; Murakoshi, K. J. Phys. Chem. C 2016, 120, 16051-16058.

(16) Schietinger, S.; Barth, M.; Aichele, T.; Benson, O. Nano Lett. 2009, 9, 1694-1698.

(17) Najmaei, S.; Mlayah, A.; Arbouet, A.; Girard, C.; Léotin, J.; Lou, J. ACS Nano 2014, 8, $12682-12689$.

(18) Hecker, N. E.; Höpfel, R. A.; Sawaki, N.; Maier, T.; Strasser, G. Appl. Phys. Lett. 1999, 75, $1577-1579$.

(19) Lal, S.; Clare, S. E.; Halas, N. J. Acc. Chem. Res. 2008, 41, 1842-1851.

(20) Hobbs, R. G.; Yang, Y.; Fallahi, A.; Keathley, P. D.; De Leo, E.; Graves, W. S.; Berggren, K. K. ACS Nano 2014, 8, 11747.

(21) Dombi, P.; Hörl, A.; Rácz, P.; Márton, I.; Trügler, A.; Krenn, J. R.; Hohenester, U. Nano Lett. 2013, 13, 674-678.

(22) Krüger, M.; Schenk, M.; Hommelhoff, P. Nature 2011, 475, 78-81.

(23) Bormann, R.; Gulde, M.; Weismann, A.; Yalunin, S. V.; Ropers, C. Phys. Rev. Lett. 2010, $105,1-4$. 
(24) Wimmer, L.; Herink, G.; Solli, D. R.; Yalunin, S. V; Echternkamp, K. E.; Ropers, C. Nat. Phys. 2014, 10, 432-436.

(25) Putnam, W. P.; Hobbs, R. G.; Keathley, P. D.; Berggren, K. K.; Kärtner, F. X. Nat. Phys. 2017, 13, 335-337.

(26) Dombi, P.; Horl, A.; Racz, P.; Marton, I.; Trugler, A.; Krenn, J. R.; Hohenester, U. Nano Lett. 2013, 13, 674-678.

(27) Harutyunyan, H.; Martinson, A. B. F.; Rosenmann, D.; Khorashad, L. K.; Besteiro, L. V; Govorov, A. O.; Wiederrecht, G. P. Nat. Nanotechnol. 2015, 10, 770-774.

(28) Sundararaman, R.; Narang, P.; Jermyn, A. S.; Goddard III, W. A.; Atwater, H. A. Nat. Commun. 2014, 5, 5788.

(29) Linic, S.; Aslam, U.; Boerigter, C.; Morabito, M. Nat. Mater. 2015, 14, 567-576.

(30) Linic, S.; Christopher, P.; Ingram, D. B. Nat. Mater. 2011, 10, 911-921.

(31) Mukherjee, S.; Libisch, F.; Large, N.; Neumann, O.; Brown, L. V; Cheng, J.; Lassiter, J. B.; Carter, E. A.; Nordlander, P.; Halas, N. J. Nano Lett. 2013, 13, 240-247.

(32) Clavero, C. Nat. Photonics 2014, 8, 95-103.

(33) Zhu, D.; Bosman, M.; Yang, J. K. W. Opt. Express 2014, 22, 9809-9819.

(34) Baffou, G.; Girard, C.; Quidant, R. Phys. Rev. Lett. 2010, 104, 136805.

(35) Sun, Y.; Jaffray, D. A.; Chen, L.-Y.; Yeow, J. T. W. IEEE T Nanotechnol 2012, 11, 441443. 
(36) Volpe, G.; Noack, M.; Aćimović, S. S.; Reinhardt, C.; Quidant, R. Nano Lett. 2012, 12, 4864-4868.

(37) Dregely, D.; Neubrech, F.; Duan, H.; Vogelgesang, R.; Giessen, H. Nat. Commun. 2013, 4, 2237.

(38) Jiang, H.; Gordon, R. Plasmonics 2013, 8, 1655-1665.

(39) Hobbs, R. G.; Yang, Y.; Keathley, P. D.; Swanwick, M. E.; Velásquez-García, L. F.; Kartner, F. X.; Graves, W. S.; Berggren, K. K. Nanotechnology 2014, 25, 465304.

(40) Schweikhard, V.; Grubisic, A.; Baker, T. A.; Thomann, I.; Nesbitt, D. J. ACS Nano 2011, $5,3724-3735$.

(41) Nagel, P. M.; Robinson, J. S.; Harteneck, B. D.; Pfeifer, T.; Abel, M. J.; Prell, J. S.; Neumark, D. M.; Kaindl, R. A.; Leone, S. R. Chem. Phys. 2013, 414, 106-111.

(42) Baffou, G.; Quidant, R.; Girard, C. Appl. Phys. Lett. 2009, 94, 153109.

(43) Jermyn, A. S.; Tagliabue, G.; Atwater, H. A.; Goddard III, W. A.; Narang, P.; Sundararaman, R. Far-from-equilibrium transport of excited carriers in nanostructures https://arxiv.org/abs/1707.07060.

(44) Madsen, S. J.; Esfandyarpour, M.; Brongersma, M. L.; Sinclair, R. 2017.

(45) Seah, M. P.; Dench, W. A. Surf. Interface Anal. 1979, 1, 2-11.

(46) Duan, H.; Manfrinato, V. R.; Yang, J. K. W.; Winston, D.; Cord, B. M.; Berggren, K. K. J. Vac. Sci. Technol. B 2010, 28, C6H11.

(47) Saldin, D. K.; Spence, J. C. H. Ultramicroscopy 1994, 55, 397-406. 
(48) Akkerman, A.; Akkerman, E. J. Appl. Phys. 1999, 86, 5809-5816.

(49) Duan, H.; Winston, D.; Yang, J. K. W.; Cord, B. M.; Manfrinato, V. R.; Berggren, K. K. J. Vac. Sci. Technol. B 2010, 28, C6C58.

(50) Swanwick, M. E.; Keathley, P. D.; Fallahi, A.; Krogen, P. R.; Laurent, G.; Moses, J.; Kärtner, F. X.; Velásquez-García, L. F. Nano Lett. 2014, 14, 5035-5043.

(51) Fallahi, A.; Kärtner, F. J. Phys. B At. Mol. Opt. Phys. 2014, 47, 234015.

(52) McCord, M. A.; Pease, R. F. W. J. Vac. Sci. Technol. B 1988, 6, 293-296.

(53) Sommer, L. H. Stereochemistry, Mechanism and Silicon; an Introduction to the Dynamic Stereochemistry and Reaction Mechanisms of Silicon Centers; McGraw-Hill: New York, 1965.

(54) Kale, M. J.; Avanesian, T.; Christopher, P. ACS Catal. 2014, 4, 116-128.

(55) Li, J.; Cushing, S. K.; Meng, F.; Senty, T. R.; Bristow, A. D.; Wu, N. Nat. Photonics 2015, $9,601-607$.

(56) Ma, X.-C.; Dai, Y.; Yu, L.; Huang, B.-B. Light Sci. Appl. 2016, 5, e16017.

(57) Boerigter, C.; Aslam, U.; Linic, S. ACS Nano 2016, 10, 6108-6115.

(58) Boerigter, C.; Campana, R.; Morabito, M.; Linic, S. Nat. Commun. 2016, 7, 10545.

(59) Alizadeh, E.; Sanche, L. Chem. Rev. 2012, 112, 5578-5602.

(60) Avouris, P.; Walkup, R. E. Annu. Rev. Phys. Chem. 1989, 40, 173-206.

(61) Atkinson, S. J.; Brundle, C. R.; Roberts, M. W. Faraday Discuss. Chem. Soc. 1974, 58, 62- 
79.

(62) Takahashi, T.; Harada, Y. Bull. Chem. Soc. Jpn. 1981, 54, 81-84.

(63) Melton, C. E. J. Chem. Phys. 1972, 57, 4218-4225.

(64) Simpson, W. C.; Sieger, M. T.; Orlando, T. M.; Parenteau, L.; Nagesha, K.; Sanche, L. J. Chem. Phys. 1997, 107, 8668-8677.

(65) Kittel, C. Introduction to Solid State Physics, 6th ed.; John Wiley and Sons, 1986.

(66) Yan, L.; Wang, F.; Meng, S. ACS Nano 2016, 10, 5452-5458.

(67) Itani, T.; Yoshino, H.; Hashimoto, S.; Yamana, M.; Samoto, N.; Kasama, K. J. Vac. Sci. Technol. B 1996, 14, 4226-4228.

(68) Choueiri, R. M.; Galati, E.; Thérien-aubin, H.; Klinkova, A.; Larin, E. M.; QuerejetaFernández, A.; Han, L.; Xin, H. L.; Gang, O.; Zhulina, E. B.; Rubinstein, M.; Kumacheva, E. Nature 2016, 538, 79-83.

Table of Contents Image 


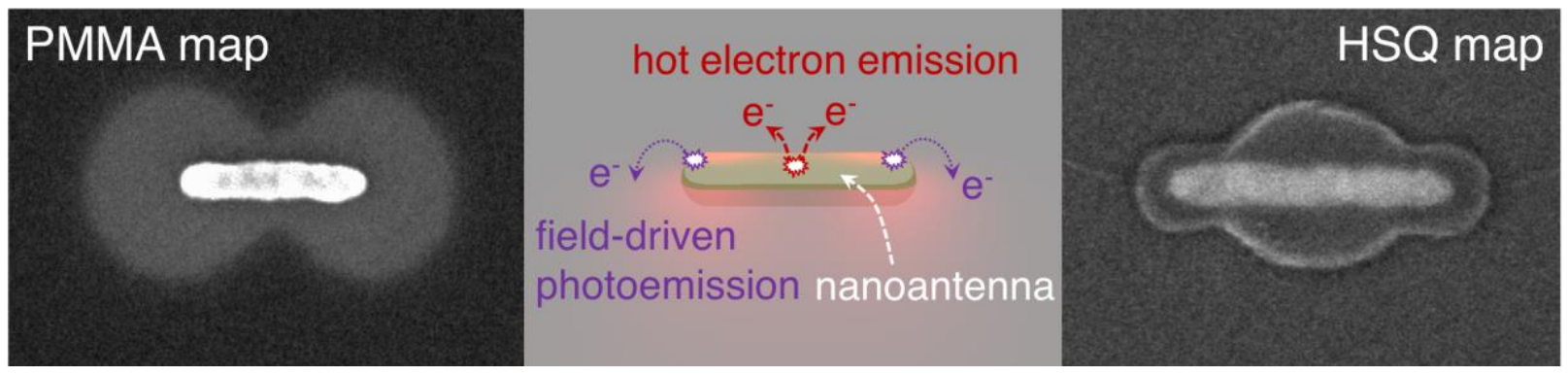

